\title{
Método GADIUS: planejando e desenvolvendo gamificaç ão adaptativa de forma dinâmica
}

Vinícius Lopes - vlopes@ inf.ufsm.br (orcid.org/0000-0002-2534-4665)

Felipe Becker Nunes - nunesfb@gmail.com (orcid.org/0000-0001-8431-7416)

Giliane Bernardi - giliane@inf.ufsm.br (orcid.org/0000-0002-6568-8921)

Roseclea Duarte Medina - rose@inf.ufsm.br (orcid.org/0000-0003-0888-6961)

Universidade Federal de Santa Maria (UFSM) - Av. Roraima n ${ }^{\circ} 1000$

Caixa Postal 5082 - CEP 97105-900 - Santa Maria - RS - Brazil

Resumo. Este artigo apresenta a descrição e avaliação do método GADIUS de gamificação adaptativa, que utiliza dados iniciais em conjunto com as interações do usuário em um ambiente educacional para promover a personalização de seus elementos de gamificação. O método visa auxiliar desenvolvedores de sistemas educacionais no processo de planejamento, desenvolvimento e aplicação de ambientes gamificados personalizáveis. O método foi avaliado por um painel de especialistas que atestou a qualidade e a utilidade de sua proposta.

Palavras-chave: gamificação, adaptaç ão, ambientes de aprendizagem.

\section{GADIUS method: plannning and developing dynamic adaptive gamification}

Abstract. This paper presents the description and evaluation of the GADIUS method for adaptive gamification. This method u ses initial u ser data, paired with u ser interactions in an educational environment, to promote its gamification e lements' personalization. It aims to assist developers of educational systems during planning, developing, and ap-plying customizable gamified environments. The method was evaluated by an expert panel, who attested to the quality and usefulness of its proposal.

Keywords: gamification, adaptation, learning environments.

\section{Introdução}

A gamificação, definida como a aplicação e uso de elementos de jogos em ambientes ex-ternos aos de um jogo [Deterding et al. 2011], vem sendo aplicada em diferentes áreas e contextos como forma de motivação e engajamento de pessoas. Em ambientes educaci-onais, a integração de elementos de gamificação é percebida c omo uma t endência emer-gente e em constante desenvolvimento [Dicheva et al. 2015]. A correta implementação desses elementos em um ambiente educacional já se provou capaz de mudar o compor-tamento de estudantes, assim como possui potencial para promover motivação e engaja-mento dos mesmos [Oliveira et al. 2018].

Recentemente, alguns autores vêm trabalhando com a ideia de personalização de ambientes gamificados, o que vem se provando uma abordagem ainda mais eficiente nes-ses ambientes [Tondello 2019]. A gamificação adaptativa usa de indicadores do usuário para personalizar o ambiente de acordo com as necessidades e preferências do usuário. Dentre os indicadores mais comuns utilizados para personalização, destacam-se idade, gênero, nível de conhecimento e perfil de jogador [Hallifax et a 1. 20 19]. No entanto, dos 
sistemas gamificados personalizáveis encontrados na literatura, a maior parte faz essa personalização apenas em uma primeira instância, muitas vezes pelo uso de questionários ou outros dados do perfil do usuário.

Poucos sistemas realizam uma automatização desse processo de personalização durante seu uso continuado pelo usuário [Toda et al. 2018]. Partindo do princípio de que um usuário pode entrar em um sistema com diferentes motivações e que as mesmas podem mudar ao longo do tempo, pode-se pressupor que a personalização feita apenas em um primeiro acesso pode ser superficial ou ineficiente [Orji et al. 20 18]. Sabendo disso, justifica-se a necessidade de desenvolver uma solução de automatização contínua do processo de personalização para ambientes gamificados, pela c arência de processos metodológicos padronizados para este tipo de cenário e pela oportunidade da formalização da gamificação adaptativa dinâmica de modo a garantir os princípios defendidos pelo próprio conceito da gamificação.

Com isso, buscou-se desenvolver um método de gamificação adaptação dinâmica para desenvolvimento de ambientes educacionais gamificados. $\mathrm{C}$ omo principal contribuição desta pesquisa, neste trabalho é apresentado o método GADIUS, que considera não apenas os dados iniciais para realizar uma adaptação estática, mas também os dados provenientes das interações do usuário com o ambiente para realizar uma adaptação dinâmica. A metodologia de concepção e desenvolvimento do método é apresentada na Seção 2, ao passo que o método em si é apresentado na Seção 3. Como processo de validação, na Seção 4 é descrita uma avaliação do método por meio de um painel com especialistas, seguida das considerações finais da pesquisa, limitações e trabalhos futuros.

\section{Metodologia}

Como primeiro passo, foi realizada uma revisão bibliográfica a fi m de compreender os conceitos oriundos da gamificação, tais como elementos, mecânicas, dinâmicas, métodos de design e aplicação, assim como arquétipos de perfil de jogador para definição de perfis de usuário. Por meio desta revisão constatou-se a necessidade de uma pesquisa mais aprofundada sobre técnicas adaptativas de gamificação, buscando m apear soluç ões de desenvolvimento de uma gamificação centrada no usuário.

Para tal, foi realizada uma revisão sistemática de literatura, relatada em [Lopes et al. 2019b], a fim de compreender como a gamificação vem sendo aplicada de forma adaptativa e personalizada em contextos educacionais e visando descobrir quais estratégias de adaptação vem sendo aplicadas em ambientes gamificados. Como principal resultado desta revisão, constatou-se a predominância de estratégias estáticas de gamificação a daptativa, onde a a daptação a o u suário o corre a penas u ma v ez, c om base em dados coletados em um primeiro momento e geralmente por meio de questionários.

Dentre esses trabalhos relacionados, destaca-se o framework $5 \mathrm{~W} 2 \mathrm{H}$, de [Klock 2017] que apresenta um ciclo de sete dimensões para o planejamento, implementação e avaliação de gamificação c entrada no u suário. O framework, todavia, não aborda aspectos de adaptação de forma dinâmica, sendo necessária a repetição do ciclo para que novas adaptações sejam feitas ao usuário. Destaca-se também, o trabalho de [Tondello 2019], onde é apresentado um método de personalização, planejamento e design de aplicações gamificadas, como foco no processo de personalização. Por si só, o método não descreve práticas que permitam a recomendação e personalização de elemen- 
tos de forma dinâmica. $\mathrm{O}$ autor sugere, todavia, que seu método pode ser incorporado a um método mais completo de planejamento e design de gamificação. Percebeu-se, portanto, a necessidade de desenvolver um método de adaptação dinâmica dos elementos de gamificação em ambientes educacionais, onde a adaptação ao usuário não se limita apenas a um primeiro momento e sim durante todo o período de utilização do ambiente pelo usuário. Iniciou-se, assim, o processo de planejamento e concepção deste método.

Para este método, como indicadores de personalização para realizar as adaptações, decidiu-se adotar o modelo Hexad [Marczewski 2015] de perfis d e j ogador. S ua escolha se justifica p or s er o único m odelo a a presentar $\mathrm{t}$ ipos $\mathrm{d}$ e $\mathrm{j}$ ogadores específicos para ambientes gamificados [Klock et al. 2016] e u m questionário e mpiricamente validado [Tondello et al. 2016]. Quanto aos elementos de gamificação, foi utilizada a lista de elementos apresentados no levantamento feito por [Tondello et al. 2017], onde tais elementos se classificam em 8 diferentes categorias de acordo com as motivações do usuário e, consequentemente, com os perfis de jogador do modelo Hexad.

Com base nas relações entre elementos de gamificação, aspectos motivacionais e perfis de jogador, a presentado no estudo de [Tondello et al. 2017], foi possível elaborar uma lista de possíveis interações derivadas dos elementos de gamificação apresentados no estudo. Essa lista, com 44 possíveis interações que um usuário pode executar com elementos de gamificação, a ssim c omo a lista de 49 e lementos d os q uais e la foi elaborada, são apresentadas durante a descrição do método GADIUS, como instrumentos metodológicos.

A cada uma das interações dessa lista foi atribuído um peso, com base nas relações dos seus elementos de origem com os perfis de jogador do modelo Hexad: onde a relação de afinidade entre o perfil de jogador com o elemento de origem da in teração era forte, foi atribuído o peso de 1 ponto, onde a relação de afinidade era moderada, foi atribuído o peso de 0,5 ponto e onde havia relação fraca foi atribuído o peso de 0,2 pontos. Após essa atribuição inicial, foram realizadas atividades adicionais para validação e refinamento desses pesos. Dentre elas, foi aplicado um questionário a usuários de ambientes gamificados com diferentes perfis e experiências com jogos, a fi m de re afirmar as relaçõe s estabelecidas pelo estudo e, consequentemente, as pontuações atribuídas. Este questionário é relatado em [Lopes et al. 2019a].

Em seguida, foi desenvolvida uma aplicação, utilizando a linguagem JavaScript e a biblioteca React.js, que permitia a inserção de usuários em um banco de dados e a simulação de interações desses usuários com os elementos de um ambiente gamificado fictício. A aplicação utilizou as métricas e instrumentos estabelecidos para o método em suas simulações, com isso foi possível simular as alterações de predominâncias dos perfis dos usuários e perceber possíveis tendências que poderiam afetar o balanceamento do sistema de adaptações. Desta forma, o peso de cada interação foi reavaliado e rebalanceado para garantir um fluxo imparcial e não tendencioso de predominância de perfis de jogador.

Após isso, foram planejadas e elaboradas as etapas do método e todos os aspectos a serem considerados em cada etapa. Com o método construído, foi realizada uma avaliação de sua qualidade por meio de um painel com especialistas de gamificação. A descrição do método, com suas etapas, aspectos e instrumentos é apresentada na Seção 3, ao passo que sua avaliação é relatada na Seção 4. 


\section{Método GADIUS}

Neste estudo, compreende-se como método uma abordagem sistemática para alcançar um objetivo ou resultado, com processos ordenados para construção de um produto ou realização de uma tarefa [IEEE 2010]. Nesse sentido, buscando desenvolver uma solução para planejamento e implementação de gamificação adaptativa de forma dinâmica, foi desenvolvido o método GADIUS, acrônimo de "Gamificação Adaptativa Dinâmica baseada nas Interações do Usuário". Este método é dividido em 4 etapas, onde são descritos diferentes aspectos e instrumentos para planejamento, construção e adaptação de um ambiente educacional gamificado (Figura 1).

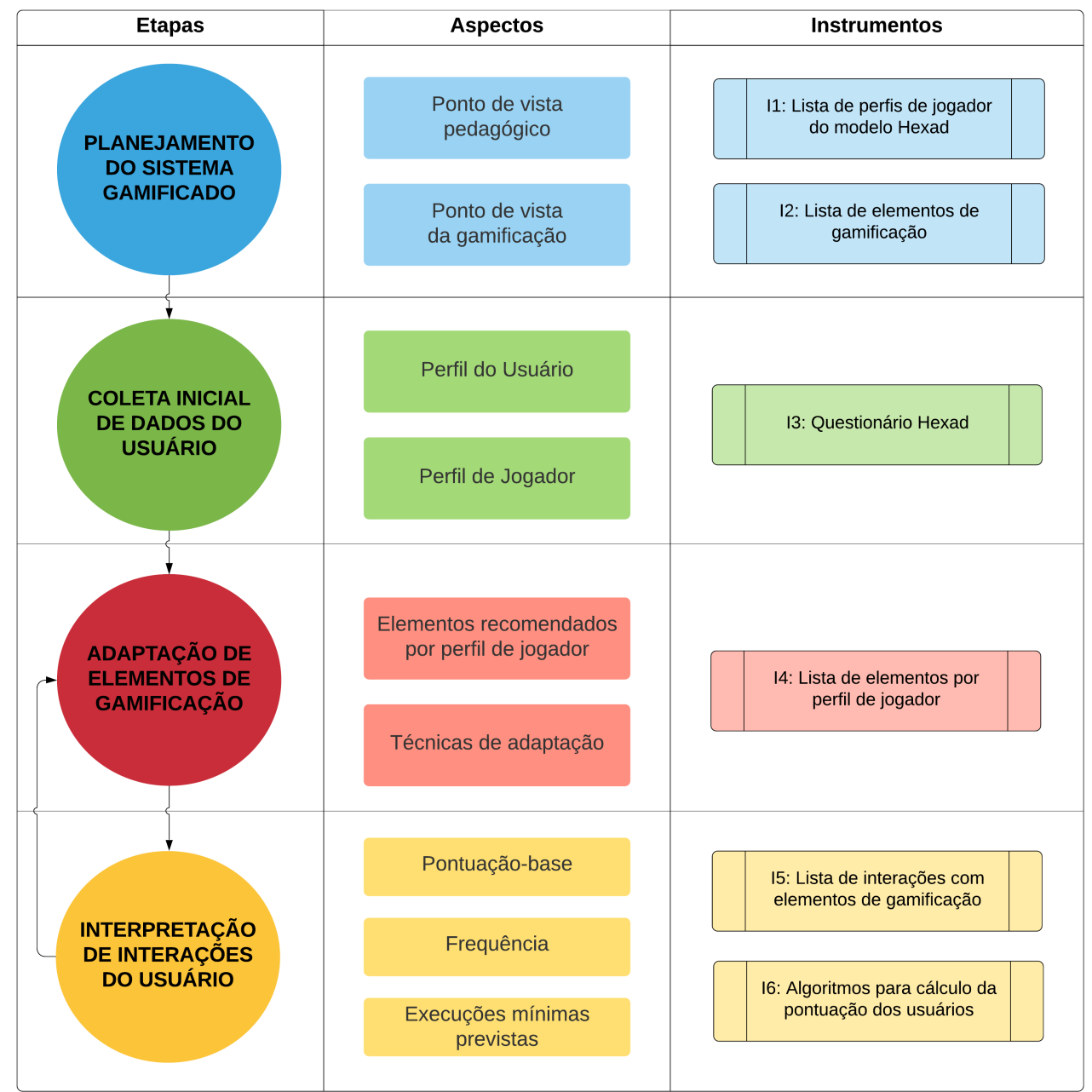

Figura 1. Método GADIUS: etapas, aspectos e instrumentos. Fonte: elaborado pelo autor.

O método é voltado para desenvolvedores de ambientes educacionais para uso em conjunto com educadores, ou para educadores com experiência em desenvolvimento desses ambientes. Busca-se, com este método, desenvolver ambientes educacionais gamificados orientados para quaisquer áreas do conhecimento.

\subsection{Planejamento do sistema gamificado}

A primeira etapa deste método corresponde aos aspectos de concepção e desenvolvimento de um ambiente de aprendizagem. Tais aspectos se referem ao planejamento do ambiente 
do ponto de vista pedagógico e do ponto de vista de sua gamificação, com o estabelecimento de quais elementos e perfis de jogador serão implementados no sistema. Quanto ao ponto de vista pedagógico, deve-se considerar informações como o público-alvo para o qual o ambiente será desenvolvido, que tipo de conteúdo será apresentado e quais são os objetivos de aprendizagem propostos. Para tal, recomenda-se a utilização dos trabalhos de [Filatro and Bileski 2017] e [Klock 2017].

Quanto ao ponto de vista da gamificação do ambiente, o desenvolvedor, em conjunto com o educador, deve selecionar quais elementos de gamificação e quais perfis de jogador o ambiente irá abranger, podendo ser todos ou apenas alguns. Para isso, devem ser analisadas a lista de perfis de jogador (Instrumento 1 - Tabela 1) e a lista de elementos de gamificação (Instrumento 2). Por elementos de gamificação compreende-se, por exemplo, missões, avatares, medalhas ou conquistas. A lista completa destes elementos pode ser acessada pelo link [bit.ly/2TBgwsC]

Tabela 1. Instrumento 1: Perfis de jogador do modelo Hexad, com suas motivações e características. Fonte: [Marczewski 2015].

\begin{tabular}{|c|c|c|}
\hline Perfil de jogador & Motivação & Características \\
\hline $\begin{array}{c}\text { Conquistador } \\
\text { (Achiever) }\end{array}$ & Domínio & Realiza-se ao superar desafios e conquistar objetivos. \\
\hline $\begin{array}{l}\text { Socializador } \\
\text { (Socialiser) }\end{array}$ & Relacionamentos & Prefere interagir e se relacionar com outros jogadores. \\
\hline $\begin{array}{c}\text { Filantropo } \\
\text { (Philantropist) }\end{array}$ & Propósito & Satisfaz-se ao ajudar os outros de forma intrínseca, altruísta. \\
\hline $\begin{array}{c}\text { Espírito Livre } \\
\text { (Free Spirit) }\end{array}$ & Autonomia & Busca liberdade para explorar um ambiente e criar coisas novas a partir dele. \\
\hline $\begin{array}{c}\text { Disruptor } \\
\text { (Disruptor) }\end{array}$ & Mudanças & Gosta de contrariar as regras para observar as consequências. \\
\hline $\begin{array}{l}\text { Jogador } \\
\text { (Player) }\end{array}$ & Recompensas & Cumpre ações e tarefas de forma extrínseca, para receber algo em troca. \\
\hline
\end{tabular}

\subsection{Coleta inicial de dados do usuário}

Após o planejamento e desenvolvimento do ambiente gamificado, o usuário terá um primeiro contato com o sistema, onde devem ser coletados os dados demográficos, experiências e habilidades a fim de traçar seu perfil de usuário, assim como também responder o questionário Hexad (Instrumento 3), elaborado por [Tondello et al. 2016], para estabelecer quais são seus perfis de jogador predominantes. O questionário pode ser acessado pelo link [bit.ly/3mzR9DZ]. Com base nas respostas do questionário, obtém-se uma pontuação do usuário para cada um dos 6 perfis de jogador do modelo Hexad e, com isso, o sistema do ambiente educacional já pode fazer suas primeiras adaptações ao usuário.

\subsection{Adaptação de elementos de gamificação}

Nesta etapa são considerados os processos de adaptação que o sistema realiza, com base nos dados iniciais do usuário e, eventualmente, pela interpretação de suas interações. Primeiramente deve-se considerar quais elementos de gamificação s ão recomendados para cada um dos perfis d e j ogador d o m odelo $\mathrm{H}$ exad. O I nstrumento 4 apresenta, na Tabela 2, recomendações de elementos para cada perfil, c om b ase $\mathrm{n}$ os e studos de [Tondello et al. 2017] e nas relações estabelecidas durante a construção do método. Com isso, o sistema é capaz de implementar suas técnicas de adaptação. O método GADIUS prevê duas técnicas de adaptação: a personalização e a recomendação. 


\begin{tabular}{|c|c|}
\hline Perfil de Jogador & Elementos Recomendados \\
\hline $\begin{array}{l}\text { Conquistador } \\
\text { (Achiever) }\end{array}$ & $\begin{array}{l}\text { Desafios, batalhas de chefe, coleções, missões, níveis, } \\
\text { medalhas ou conquistas, recompensas ou prêmios. }\end{array}$ \\
\hline $\begin{array}{l}\text { Espírito Livre } \\
\text { (Free Spirit) }\end{array}$ & $\begin{array}{l}\text { Avatar, customização, easter eggs, tema, narrativa ou história, } \\
\text { tarefas de exploração, conteúdo raro ou desbloqueável. }\end{array}$ \\
\hline $\begin{array}{l}\text { Socializador } \\
\text { (Socialiser) }\end{array}$ & $\begin{array}{l}\text { Descoberta social, redes sociais, trocas convites de amigos, } \\
\text { status social, equipes ou guildas, placares de líderes. }\end{array}$ \\
\hline $\begin{array}{l}\text { Disruptor } \\
\text { (Disruptor) }\end{array}$ & $\begin{array}{l}\text { Placares de líderes, competição social, acesso, easter eggs, } \\
\text { comparação ou pressão social, loterias ou jogos de chance. }\end{array}$ \\
\hline $\begin{array}{l}\text { Jogador } \\
\text { (Player) }\end{array}$ & $\begin{array}{l}\text { Certificados, coleções, missões, recompensas ou prêmios, economia } \\
\text { virtual, medalhas ou conquistas, feedback de progressão. }\end{array}$ \\
\hline $\begin{array}{c}\text { Filantropo } \\
\text { (Philantropist) }\end{array}$ & $\begin{array}{l}\text { Compartilhamento de conhecimento, presentes, aprendizado, mecanismos de } \\
\text { votação, papéis administrativos, ferramentas de criatividade e desenvolvimento. }\end{array}$ \\
\hline
\end{tabular}

Na personalização, ocorrem alterações diretas à interface do ambiente, mudando a ordem e priorizando a organização dos elementos com base nos perfis de jogador predominantes do usuário (com maior pontuação). A recomendação, por sua vez, vai no sentido oposto, utilizando-se de notificações, sugestões e mensagens para apresentar ao usuário elementos referentes aos seus perfis não predominantes (com menor pontuação), visando uma homogeneidade nas possibilidades de interações do usuário. A Figura 2 apresenta duas telas de exemplos de interface com possibilidades de personalização e recomendação. Na esquerda, um ambiente personalizado para socializadores e na direita um ambiente personalizado para conquistadores, com recomendação de interações de um perfil socializador.
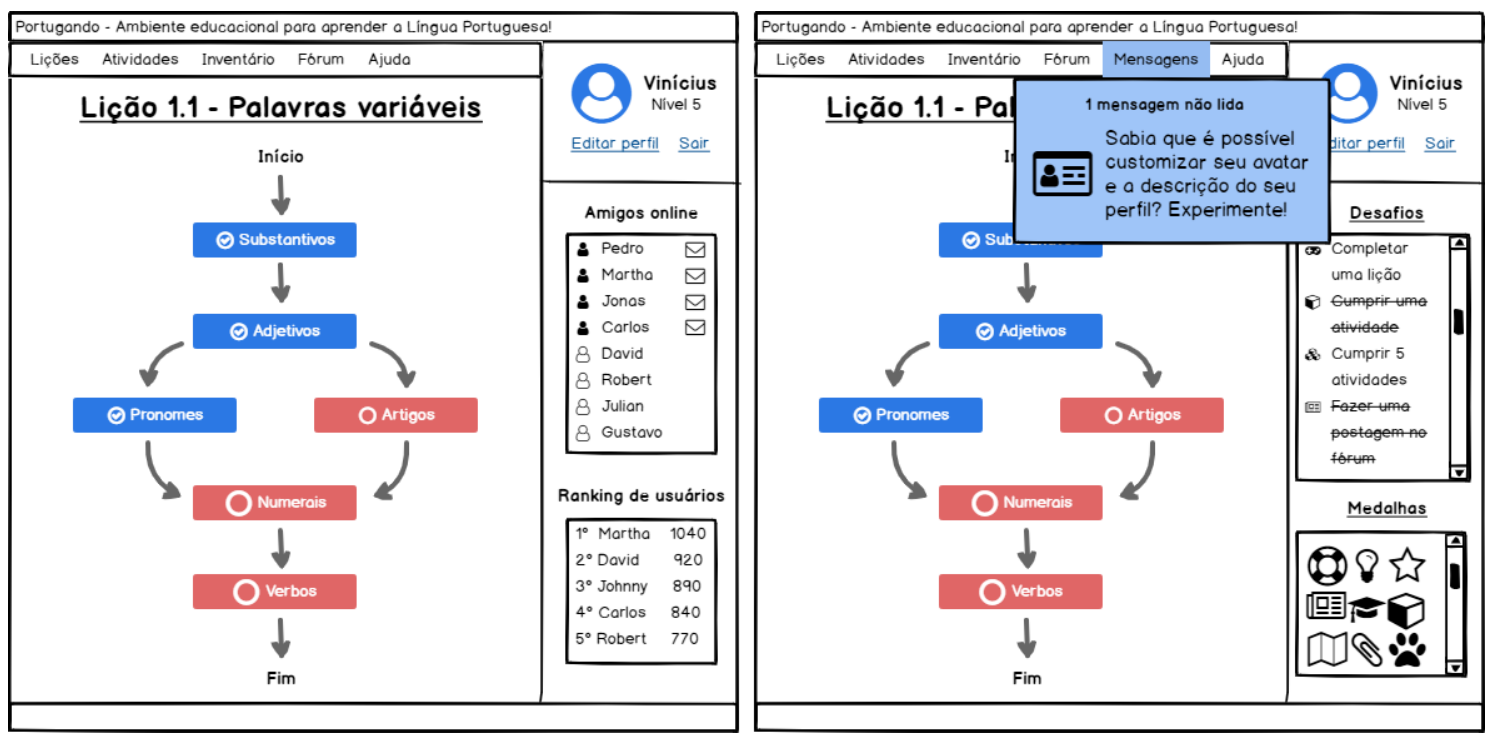

Figura 2. Exemplos de personalização e recomendação em ambiente gamificado fictício. Fonte: elaborado pelo autor.

O momento em que a adaptação do ambiente deve ocorrer depende do planejamento do desenvolvedor, de acordo com as necessidades e escopo do ambiente educacional, podendo acontecer a cada nova entrada do usuário no sistema, ou dentro de um período fixo $\mathrm{p}$ ré-estabelecido. P orém, a i nterpretação $\mathrm{d}$ as interaç ões d o u suário e contabilização dentro do sistema deve ocorrer a cada ciclo de acesso do usuário ao ambiente. Para tal, compreende-se como ciclo de acesso os processos de entrada, navegação e saída do usuário dentro do ambiente. 


\subsection{Interpretação de interações do usuário}

Para contabilizar as interações do usuário com o ambiente gamificado, foi desenvolvida uma lista com 44 possíveis interações com elementos de gamificação, com um peso sobre cada interação para cada um dos 6 perfis de jogador do modelo Hexad. Na Tabela 3 são apresentados alguns exemplos de interações dessa lista, compreendida neste método como o Instrumento 5. A lista completa de interações com elementos de gamificação pode ser acessada pelo link [bit.ly/3mE1pLg].

Tabela 3. Instrumento 5: Lista parcial de interações com sua pontuação-base
para cada perfil de jogador. Fonte: Elaborado pelo autor.
\begin{tabular}{clcccccc} 
Cód. & Interação & ACH & FRS & SOC & DIS & PLA & PHI \\
\hline I01 & Abrir postagens/mensagens de outros usuários & 0,5 & 0 & 1 & 0 & 0,5 & 0,2 \\
I02 & Abrir presentes recebidos dentro do jogo & 0,5 & 0 & 0,5 & 0 & 0,2 & 1 \\
I03 & $\begin{array}{l}\text { Aceitar/cumprir uma missão oferecida por outro } \\
\text { usuário ou pelo sistema }\end{array}$ & 0 & 0 & 0 & 0 & 1 & 0 \\
I04 & $\begin{array}{l}\text { Acessar a pontuação de outros usuários } \\
\text { Acessar alguma tela para verificar seu progresso } \\
\text { no sistema }\end{array}$ & 0,5 & 0 & 1 & 0 & 0,5 & 0 \\
I05 & 0,2 & 0 & 0 & 0 & 0,2 & 0,2
\end{tabular}

Além da pontuação-base estabelecida por esses pesos atribuídos, utilizam-se outras variáveis, como frequência de execuções e execuções mínimas previstas de uma interação por acesso. Com essas variáveis, é possível calcular, a cada ciclo de acesso, a pontuação do usuário para cada perfil de jogador, e com isso determinar quais são seus perfis predominantes e não predominantes. A Figura 3 apresenta, como Instrumento 6, um algoritmo utilizando todas as variáveis consideradas nesse processo.

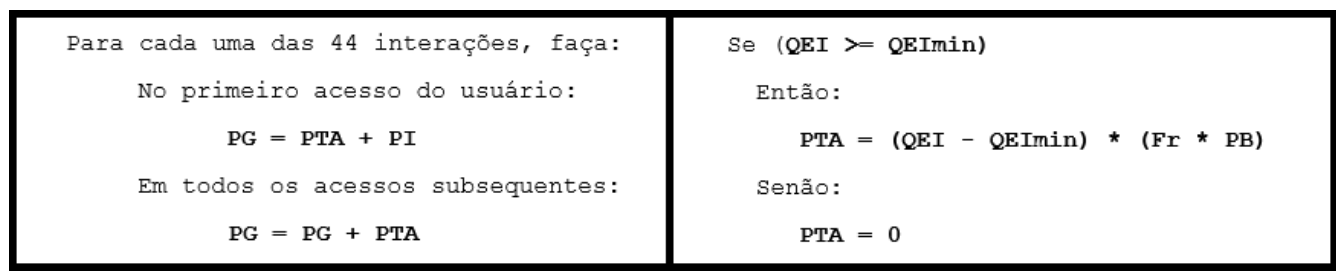

Figura 3. Instrumento 6: Algoritmo para cálculo das pontuações de interações do usuário. Fonte: elaborado pelo autor.

Um maior detalhamento dessas variáveis, assim como a descrição completa e detalhada do método GADIUS é acessível em [Lopes 2020].

\section{Avaliação do método com especialistas}

Para avaliar a qualidade do método no cumprimento de sua proposta, foi realizado um painel com especialistas, que consiste na consulta a diversos especialistas de uma área específica a fi m de obter su a op inião so bre as pectos es pecíficos de uma pesquisa [Beecham et al. 2005]. Para tal, foi selecionado um painel com 46 especialistas em gamificação, dentre d outores, pós-doutores ou doutorandos com p rojetos e publicações dentro dessa área. A esses especialistas foi enviado um questionário virtual, com a descrição formal do método GADIUS, acompanhado de perguntas avaliativas sobre as características de qualidade do método. As perguntas enviadas foram estruturadas a partir da abordagem GQM (Goal, Question, Metric) de [Basili 1994], onde foram elaboradas métricas para responder questões de pesquisa a respeito de características de qualidade do método GADIUS. Foram utilizadas as características de qualidade comumente utilizadas na avaliação modelos e processos [Rittgen 2010]. 
O questionário ficou aberto por um período de 30 dias, em julho de 2020, obtendo uma adesão de 20 especialistas. A descrição completa e detalhada desta avaliação está presente em [Lopes 2020]. A Figura 4 apresenta um resumo das avaliações desses especialistas, com sua opinião a respeito da existência de características específicas de qualidade do método GADIUS, como autenticidade, flexibilidade, validade e usabilidade. Tais características foram avaliadas em uma escala Likert de 5 pontos, de discordo a concordo. Em geral, constatou-se uma visão positiva dos especialistas quanto a presença das características avaliadas, com destaque para a autenticidade e utilidade do método.

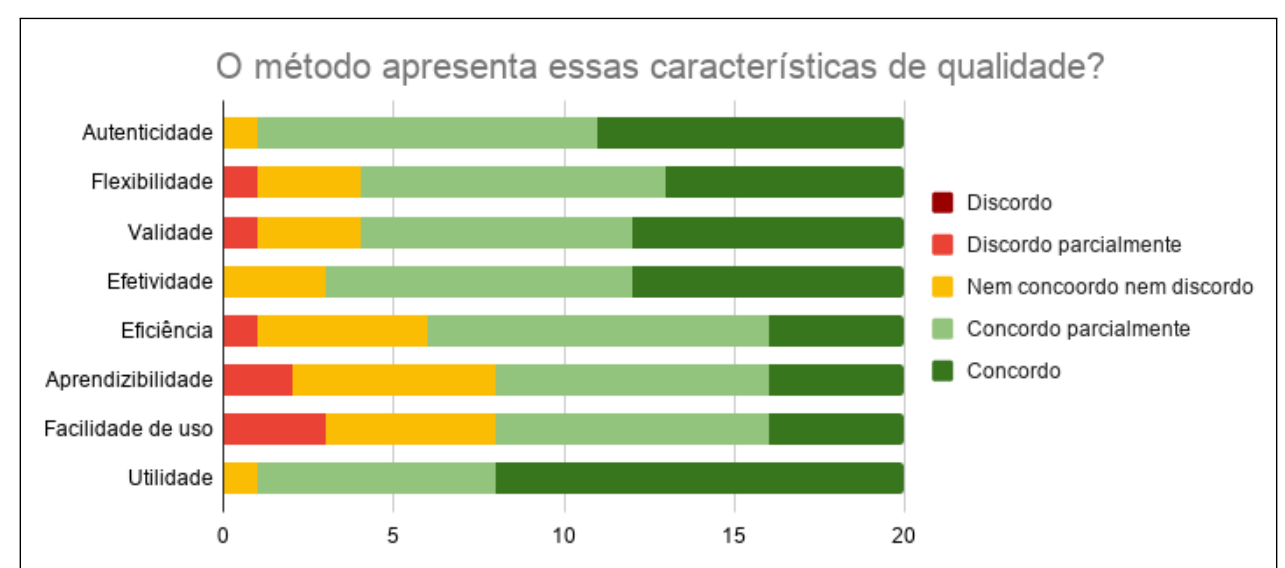

Figura 4. Avaliação dos especialistas de autenticidade, flexibilidade, validade e características de usabilidade. Fonte: elaborado pelo autor.

Na Figura 5, por sua vez, é apresentada a percepção dos especialistas quanto a ausência de características de qualidade como completude, compreensibilidade, consistência, correção e não ambiguidade do método. Foi realizada uma pergunta para cada característica, com alternativas de 'sim', 'não' e 'não tenho condições de responder' como resposta e um espaço para justificativas e observações.

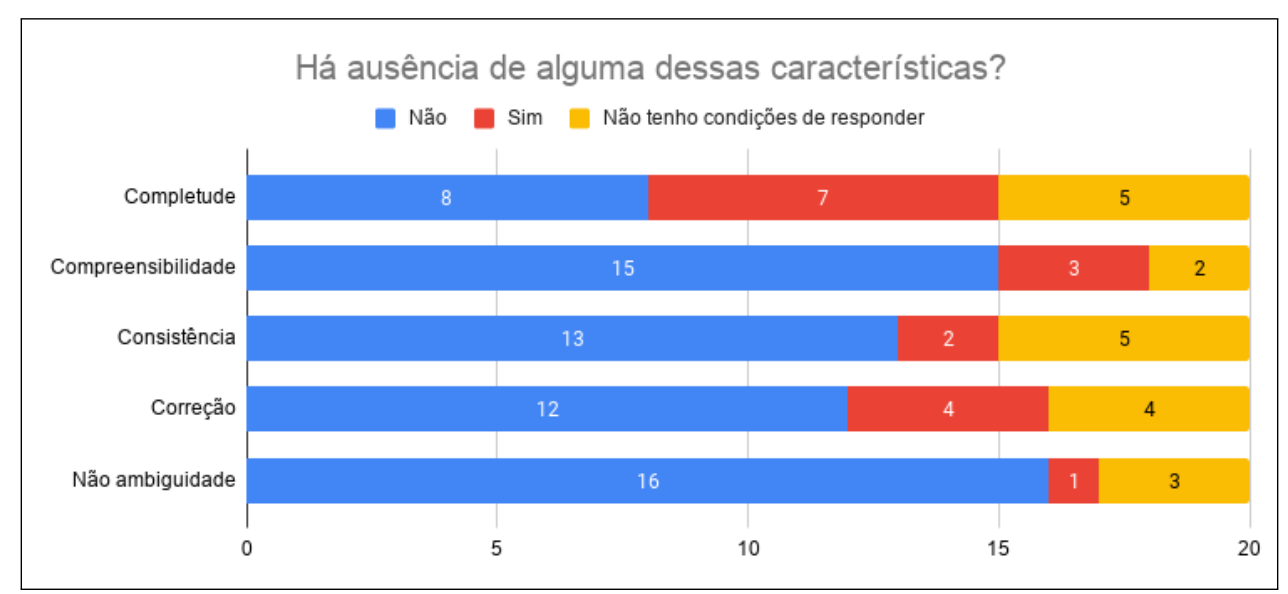

Figura 5. Avaliação dos especialistas de completude, compreensibilidade, consistência, correção e não ambiguidade. Fonte: elaborado pelo autor.

Em sua maioria, os especialistas não identificaram a ausência das características questionadas. Alguns especialistas preferiram se abster dessas questões, justificando falta de experiência com o desenvolvimento de ambientes educacionais e todos os aspectos envolvidos no processo. Dentre os principais comentários dos especialistas, destacouse a ausência da aplicação do método em cenários mais complexos e a necessidade de 
exemplos adicionais, seja pela aplicação em ambientes simulados ou em cenários reais. Foi apontada também a falta de clareza na descrição de algumas etapas do método, com alguns comentários apresentando sugestões para melhoria de algumas características, com base nas fragilidades apontadas. Tais sugestões foram adotadas e já se encontram presentes na versão atual do método GADIUS. Com base na opinião dos especialistas, constatou-se que, em geral, o método possui um bom potencial no cumprimento do seu objetivo, atendendo os requisitos mínimos para o planejamento, implementação e execução de gamificação adaptativa de forma dinâmica em ambientes educacionais.

\section{Considerações Finais}

Com a popularização da gamificação em ambientes educacionais como técnica para promover o ensino-aprendizagem, muito tem se discutido a respeito de técnicas de personalização e adaptação desses ambientes, a fim de otimizar a motivação e o engajamento dos estudantes. Com base nisso, este trabalho descreveu a concepção, desenvolvimento e avaliação do método GADIUS, para aplicação de gamificação adaptativa de forma dinâmica em ambientes educacionais. O método GADIUS foi desenvolvido com o objetivo de auxiliar desenvolvedores de ambientes educacionais no processo de planejamento, desenvolvimento e aplicação de gamificação adaptativa de forma dinâmica. Espera-se que, um ambiente gamificado utilizando o método GADIUS possa atender diferentes perfis, promovendo maior motivação e engajamento de seus usuários.

O método foi avaliado por um painel de especialistas em gamificação, onde reconhece-se que o mesmo representa uma contribuição significativa na área da gamificação, apresentando uma boa viabilidade de sucesso em sua proposta. Dentre as limitações percebidas nesta pesquisa, destaca-se a ausência da aplicação do método GADIUS em cenários mais complexos, tais como aplicações-teste e ambientes simulados. Percebe-se a carência de avaliações mais robustas, por meio da análise de dados oriundos de interações e do feedback de usuários reais. Para trabalhos futuros, planeja-se o desenvolvimento de um ambiente gamificado, desenvolvido a partir do método GADIUS. Com isso, almeja-se coletar dados de interações e feedback de usuários reais, a fim de realizar novas avaliações de qualidade do método sob o ponto de vista de desenvolvedores e de usuários de ambientes educacionais.

\section{Referências}

Basili, V. R. (1994). Software modeling and measurement: the goal/question/metric paradigm. Technical report.

Beecham, S., Hall, T., Britton, C., Cottee, M., and Rainer, A. (2005). Using an expert panel to validate a requirements process improvement model. Journal of Systems and Software, 76(3):251-275.

Deterding, S., Dixon, D., Khaled, R., and Nacke, L. (2011). From game design elements to gamefulness: defining gamification. In Proceedings of the 15th international academic MindTrek conference, pages 9-15. ACM.

Dicheva, D., Dichev, C., Agre, G., Angelova, G., et al. (2015). Gamification in education: A systematic mapping study. Educational Technology \& Society, 18(3):75-88.

Filatro, A. C. and Bileski, S. C. (2017). Produção de conteúdos educacionais. Saraiva Educação SA. 
Hallifax, S., Serna, A., Marty, J.-C., and Lavoué, É. (2019). Adaptive gamification in education: A literature review of current trends and developments. In European Conference on Technology Enhanced Learning, pages 294-307. Springer.

IEEE (2010). Iso/iec/ieee international standard - systems and software engineering vocabulary. ISO/IEC/IEEE 24765:2010(E), pages 1-418.

Klock, A. C. T. (2017). Análise da influência da gamificação na interação, na comunicação e no desempenho dos estudantes em um sistema de hipermídia adaptativo educacional.

Klock, A. C. T., Flores, T. H., Gasparini, I., and HOUNSELL, M. d. S. (2016). Classificação de jogadores: um mapeamento sistemático da literatura. Anais do Simpósio Brasileiro de Jogos e Entretenimento Digital. Porto Alegre: SBC.

Lopes, V. (2020). Gadius: Um método para gamificação adaptativa dinâmica baseada em interações do usuário.

Lopes, V., Medina, R., Bernardi, G., and Nunes, F. B. (2019a). Um modelo conceitual para adaptação contínua de elementos de gamificação em ambientes educacionais. In Brazilian Symposium on Computers in Education (Simpósio Brasileiro de Informática na Educação-SBIE), volume 30, page 992.

Lopes, V., Reinheimer, W., Bernardi, G., Medina, R., and Nunes, F. B. (2019b). Adaptive gamification strategies for education: a systematic literature review. In Brazilian Symposium on Computers in Education (Simpósio Brasileiro de Informática na EducaçãoSBIE), volume 30, page 1032.

Marczewski, A. (2015). Even Ninja Monkeys Like to Play: Gamification, Game Thinking and Motivational Design. CreateSpace Independent Publishing Platform.

Oliveira, W. d. S., Toda, A., Isotani, S., and Bittencourt, I. I. (2018). Does gamified educational systems change students' learning behaviors? a case study with postgraduate students. RENOTE-Revista Novas Tecnologias na Educação, 16(2):11-20.

Orji, R., Tondello, G. F., and Nacke, L. E. (2018). Personalizing persuasive strategies in gameful systems to gamification user types. In Proceedings of the $2018 \mathrm{CHI}$ Conference on Human Factors in Computing Systems, page 435. ACM.

Rittgen, P. (2010). Quality and perceived usefulness of process models. In Proceedings of the 2010 ACM Symposium on Applied Computing, pages 65-72.

Toda, A. M., dos Santos, W. O., Klock, A. C., Gasparini, I., Bittencourt, I. I., and Isotani, S. (2018). Frameworks para o planejamento da gamificação em contextos educacionais-uma revisão da literatura nacional. RENOTE - Novas Tecnologias na Educação, 16(2).

Tondello, G. F. (2019). Dynamic personalization of gameful interactive systems.

Tondello, G. F., Mora, A., and Nacke, L. E. (2017). Elements of gameful design emerging from user preferences. In Proceedings of the Annual Symposium on Computer-Human Interaction in Play, pages 129-142. ACM.

Tondello, G. F., Wehbe, R. R., Diamond, L., Busch, M., Marczewski, A., and Nacke, L. E. (2016). The gamification user types hexad scale. In Proceedings of the 2016 annual symposium on computer-human interaction in play, pages 229-243. ACM. 